\title{
Magic number behavior for heat capacities of medium sized classical Lennard-Jones clusters
}

\author{
D. D. Frantz \\ Department of Chemistry, University of Waterloo, \\ Waterloo, Ontario N2L 3G1, Canada
}

(October 24, 2018)

\begin{abstract}
Monte Carlo methods were used to calculate heat capacities as functions of temperature for classical atomic clusters of aggregate sizes $25 \leq N \leq 60$ that were bound by pairwise Lennard-Jones potentials. The parallel tempering method was used to overcome convergence difficulties due to quasiergodicity in the solid-liquid phase-change regions. All of the clusters studied had pronounced peaks in their heat capacity curves, most of which corresponded to their solid-liquid phase-change regions. The heat capacity peak height and location exhibited two general trends as functions of cluster size: for $N=25$ to 36 , the peak temperature slowly increased, while the peak height slowly decreased, disappearing by $N=37$; for $N=30$, a very small secondary peak at very low temperature emerged and quickly increased in size and temperature as $N$ increased, becoming the dominant peak by $N=36$. Superimposed on these general trends were smaller fluctuations in the peak heights that corresponded to "magic number" behavior, with local maxima found at $N=36,39,43,46$ and 49, and the largest peak found at $N=55$. These magic numbers were a subset of the magic numbers found for other cluster properties, and can be largely understood in terms of the clusters' underlying geometries. Further insights into the melting behavior of these clusters were obtained from quench studies and by examining rms bond length fluctuations.
\end{abstract}




\section{INTRODUCTION}

The investigation of small atomic and molecular clusters remains an active area of resparch, both theoreticallyt 13 and experimentally.14 17 Computer simulations of clusters have revealed a wide diversity of fundamental behavior that is highly sensitive to many factors, such as the cluster aggregate size $N$, the potential used to model the intermolecular interactions, and even the simulation method itself. The Lennard-Jones (LJ) potential is the most commonly used model potential for computer simulations of cluster properties, and it is especially useful for modeling rare gas clusters. A key aspect of this model when applied to small and medium sized atomic clusters is that the resulting potential surfaces have global minima that are dominated by icosahedral-like structures, with fivefold symmetries that are markedly different than the closest-packed structure found in corresponding bulk systems.

A major motivation for studying clusters is the insight that can be obtained in understanding the transition from finite to bulk behavior. Because of their high proportion of surface atoms, many clusters have physical properties that typically exhibit a very irregular dependence on their aggregate size, with certain sizes standing out in particular. For example, the mass spectra of clusters typically exhibit especially abundant sizes that often reflect particularly stable structures, specially reactive clusters, or closed electronic shells $12,14,17$ These "magic pymber" sizes have been of great theoretical interest, $11-13$ and much work has been done in relating magic number sequences to cluster structure in terms of "soft" sphere packings, since many magic number sizes in LJ clusters such as $N=13,19,55$, and 147 correspond to compact structures that are especially stable. 10 Many cluster properties obtained from simulation studies such as binding energies, root mean square (rms) bond length fluctuations and heat capacities also show very irregular dependencies on the cluster size that mostly correspond to their usual magic number sequences. For exam ple, the heatcanacity peak values per atom for $\mathrm{LJ}_{13} 3,21$ and $\mathrm{LJ}_{55} 25,35-37$ are especially large, which can be attributed to the exceptional thermodynamic stability of their lowest-energy isomers relative to their next higher energy configurations. To more easily compare various cluster property size dependencies in this report, I have generalized the concept of magic numbers for arbitrary cluster properties to refer to those cluster sizes having particularly enhanced values relative to their immediate neighbors. In a previous study, I examined these generalized magic number effects in LJ clusters ranging in size from 4 to 24 atoms 11 In this study, I extend the sequence to 60 atoms, which completes the filling of the second Mackay icosahedral shell (to $N=55$ ) and just begins the filling of the third shell (to $N=147$ ).

The issue of cluster phase transitions is fundamentally important, and so has been of great interest. Because of their small size, clusters do not have the sharp first order transition characteristic of bulk melting, but instead show changes occurring over a range of temperatures, typically a few degrees $\mathrm{K}$ Although the phasechange range generally decreases as the cluster size increases, coalescing to the bulk transition temperature as $N$ approaches infinity, both the range and the location show much variation as a function of $N$. Cluster solid-liquid phase-change regions can be determined from computer simulations typically by identifying characteristic changes in certain cluster properties. For example, cluster potential energies often change markedly as functions of temperature in the phase-change regions, resulting in distinct peaks in the corresponding heat capacity curves; rms bond length fluctuations often rise abruptly with increasing temperature at the beginning of the phase-change regions, and the fractions of quenched cluster configurations corresponding to the lowest-energy isomers often decrease sharply with increasing temperature in these regions. Defining the phase-change region on the basis of such behavior is somewhat ambiguous, though, since the temperature ranges associated with these different properties do not always coincide, and occasionally do not even overlap. For a given property, the phase-change region temperature range can also depend on the simulation method; for example, for $\mathrm{Ar}_{13}$, the rise in the rms bond length fluctuations as calculated from canonical Monte Carlo simulations occurred about $7 \mathrm{~K}$ lower than that calculated from molecular dynamics simulations. 3 Because of these difficulties, it is convenient to loosely define the solid-liquid phase-change region as the temperature region between the lower-temperature, solid-like region (where the clusters are mostly confined to their lower energy configurations, undergoing isomerizations only rarely at the higher-temperature end of the region) and the higher-temperature, liquid-like region (where the clusters exhibit easy isomerization and diffusion). Cluster behavior in the solid-liquid phasechange regions is particularly complicated, with some cluster sizes showing coexistence behavior, where "solid like" and "liquid-like" isomers dynamically coexist, 2, 4 while other cluster sizes show a relatively smooth progression of isomerizations between locally similar but globally distinct configurations occurring throughout this region.5 Although clusters having magic number sizes such as 13 and 55 have pronounced heat capacity peaks that correspond closely to the solid-liquid phase-change region determined from other cluster properties, other cluster sizes such as $N=15,16$ and 17 do not have heat capacity peaks in their phase-change regions.11 Since heat capcity peaks haye often heen used to identify phasechange regions 20,25,32,33 35,39 characterizing their temperature dependencies for sequences of LJ clusters can help elucidate the underlying reasons for such varied behavior.

The accurate determination of cluster properties such as the heat capacity is hampered by the poor convergence of standard simulation methods in the phase-change re- 
gions. This poor convergence is a consequence of quasiergodicity, or the incomplete sampling of configuration space.18 Various methods have been developed in recent years to reduce the systematic errors resulting from quasiergodicity, including histogram methods, 20 jumpwalking methods (J-walking) 2128 smart walking methods (S-walking), 29 and parallel tempering methods. $30-32$ Many of these methods are based on the coupling of configurations obtained from ergodic higher-temperature simulations to the quasiergodic lower-temperature simulations. In my previous study of magic number effects in cluster heat capacities, I used J-walking to successfully overcome quasiergodicity. Monte Carlo J-walking methods couple the usual small-scale Metropolis moves made by a lower-temperature random walker, with occasional large-scale jumps that move the random walker out of the confined regions of configuration space that it could not otherwise easily escape within the duration of the simulation. There are two complimentary implementations of J-walking. In tandem J-walking, higher-temperature walkers and lower-temperature walkers are run in tandem, with the higher-temperature walkers occasionally feeding configurations to the lower-temperature walkers. Because J-walking obeys detailed balance only approximately, the walkers become correlated whenever a lowertemperature walker accepts a jump, and so it is necessary to run several extra Metropolis passes after a jump is accepted to break these correlations, thereby decreasing the overall efficiency. The second method breaks the correlations by running the higher-temperature walkers beforehand, and storing representative samples of the configurations in external files, which the lower-temperature walkers sample periodically in a random fashion. Thus, sampling from an external distribution is more efficient, provided that the required distributions are not too large, and this was the implementation I used in the previous study. It was also successful for the first few clusters considered in this study, but for clusters ranging in size from $N=31$ to 37 , which had two heat capacity peaks, the heat capacity curves obtained with different J-walker distributions showed poor reproducibility for the lowertemperature peaks. Since the distribution file sizes already totalled more than $2 \mathrm{~Gb}$, it seemed prudent to abandon that implementation and switch to the tandem J-walker implementation. However, a recent study by Neirotti, et al.22 of $\mathrm{LJ}_{38}$, which is notoriously difficult to simulate accurately, showed that the parallel tempering method was remarkably successful in overcoming quasiergodicity and calculating an accurate heat capacity curve. Parallel tempering is nearly identical to a parallel version of tandem J-walking, except that instead of copying the higher-temperature J-walker's configuration to the lowertemperature walker's configuration whenever a jump is accepted, the two configurations are exchanged. This has the advantage of maintaining detailed balance, thereby eliminating the need to run extra Metropolis passes after accepting an exchange, which makes the method more efficient than tandem J-walking. Thus, I decided to use parallel tempering for all of the cluster sizes examined in this study.

I begin in Section II with a brief description of the computational methods used in this work and a summary of the calculations undertaken. Section III] describes the results obtained for the cluster potential energies, heat capacities, rms bond length fluctuations and quenched configuration distributions, as functions of temperature and cluster size. In Section IV, I summarize the important conclusions concerning magic number behavior in LJ clusters for $N \leq 60$.

\section{COMPUTATIONAL METHODS}

The clusters studied were modeled by the pairwise additive Lennard-Jones potential,

$$
\begin{aligned}
V & =\sum_{i<j} V_{L J}\left(r_{i j}\right), \\
V_{L J}\left(r_{i j}\right) & =4 \epsilon\left[\left(\frac{\sigma}{r_{i j}}\right)^{12}-\left(\frac{\sigma}{r_{i j}}\right)^{6}\right] .
\end{aligned}
$$

Because small clusters can dissociate at higher temperatures and make the cluster definition ambiguous, it is customary to confine the clusters by a constraining potential. For classical Monte Carlo simulations, a perfectly reflecting spherical constraining potential of radius $R_{c}$, centered on the cluster's center of mass, is most convenient, and was used in this work.

The classical internal energy and heat capacity were calculated by the usual expressions for an $N$-atom cluster (in reduced units),

$$
\begin{aligned}
\left\langle U^{*}\right\rangle & =\frac{3 N T^{*}}{2}+\left\langle V^{*}\right\rangle, \\
\left\langle C_{V}^{*}\right\rangle & =\frac{3 N}{2}+\frac{\left\langle\left(V^{*}\right)^{2}\right\rangle-\left\langle V^{*}\right\rangle^{2}}{\left(T^{*}\right)^{2}},
\end{aligned}
$$

where $U^{*}=U / \epsilon, V^{*}=V / \epsilon, C_{V}^{*}=C_{V} / k_{B}$, and $T^{*}=$ $k_{B} T / \epsilon$. Since so many rare gas cluster simulations have been done for Ar, I found it convenient to generate the temperature-dependent curves using Ar parameters, with $\sigma=3.405 \AA$ and $\epsilon=119.4 \mathrm{~K}$.

Relative rms bond length fluctuations have been culated in many previous cluster simulations.2 20.11 .38 , 39 These were calculated as

$$
\delta=\frac{2}{N(N-1)} \sum_{i<j} \frac{\left(\left\langle r_{i j}^{2}\right\rangle-\left\langle r_{i j}\right\rangle^{2}\right)^{1 / 2}}{\left\langle r_{i j}\right\rangle},
$$

where the summations are taken over the entire walk. Because of the summations being taken over the entire walk, rms bond length fluctuations cannot be determined for the J-walking and parallel tempering simulations, whose configuration jumps and exchanges effectively reduce the 
long Metropolis walks to a collection of very many short excursions about the last accepted jump or exchange configuration. Thus, quasi-ergodicity remains a problem for rms bond length calculations. Caution must also be exercised when interpreting $\delta(T)$ curves, since they are dependent on simulation parameters such as the walk length, as well as the ensemble that is used 3.1139

\section{A. J-walking}

J-walking runs were done for cluster sizes $25 \leq N \leq 38$, using sampling from externally stored distributions as described in the preceding study.11 Distributions for each temperature consisted of several files, totaling $2.5 \times 10^{6}$ configurations, sampled every $100,50,25$, or 10 passes, depending on the temperature (100 passes for higher temperatures having broad distributions, 10 passes for very low temperatures having very narrow distributions). The distributions were generated in stages from higher temperatures to lower, beginning with temperatures well within the cluster liquid region (50 to $60 \mathrm{~K}$ ). Data accumulation was done with walks consisting of $2 \times 10^{6}$ total passes, with jumps attempted randomly with a frequency of $10 \%$. Since any systematic errors in the higher temperature J-walker distributions can feed into the lower temperature distributions and corrupt them, heat capacity peaks occurring at very low temperatures can be very difficult to calculate accurately. In previous studies of 13-atom binary clusters, 44 very low temperature heat capacity peaks corresponding to mixing anomalies could be obtained accurately by repeating the full J-walking temperature scan several times, each time with newly generated distributions, and averaging the independent curves. This procedure was followed in this work as well, and at least five J-walking runs were done for cluster sizes 25 to 30 , with the heat capacity curves showing very good reproducibility in each case. For cluster sizes 31 to 38 though, the curves showed very poor reproducibility for the lower-temperature peaks, and so the method was abandoned.

Despite the J-walking method's inability to reproduce the lower-temperature heat capacity peaks accurately, the method was still useful for obtaining a listing of the important lowest-energy configurations for each cluster size. In each case, representative subsets of the distribu tion files were quenched using steepest descent methods 19 and all of the unique isomers found were extracted. While this crude method cannot identify all of the configurations for clusters of these sizes, it is well suited for identifying most of the lower energy isomers that dominate the low temperature behavior of clusters. For example, the quenched $\mathrm{J}$-walker distributions for $\mathrm{LJ}_{38}$ contained all of the lowest-energy configurations found by Doye et al. 34 including the global minimum face-centered-cubic (fcc) truncated octahedron, which is notoriously difficult to obtain from randomly initialized simulations. These findings imply that J-walking was successful in accessing all of the important regions of configuration space for these clusters, even though it was unable to do so in the statistically representative manner needed to accurately reproduce the heat capacities at very low temperatures. Thus, higher temperature J-walker distributions were also generated for cluster sizes 39 to 60 to obtain sets of the important low-energy quenched configurations.

\section{B. Metropolis Monte Carlo}

Metropolis simulations were also run for each cluster. These provided a check of the J-walking and parallel tempering results for those temperature regions where quasiergodicity in the Metropolis runs was not a problem, as well as revealing trends in the systematic errors arising from quasiergodicity in the Metropolis runs themselves. Temperature scans were also generated using Ar parameters, with the temperature mesh size set to $\Delta T=1 \mathrm{~K}$, and sometimes to $0.5 \mathrm{~K}$ in the phase-change regions. For each temperature, simulations consisted of $10^{5}$ warmup passes, followed by $10^{7}$ passes with data accumulation. The scans in each case were started at the lowest temperature from the global minimum configuration obtained from the corresponding J-walker simulations, and were continued well past the cluster solid-liquid phase-change region, with the final configuration for each temperature used as the initial configuration for the subsequent temperature. To gain a better appreciation of the extent of quasiergodicity in the solid-liquid phase-change region, additional Metropolis simulations consisting of $10^{8}$ total passes per temperature point were run for each cluster over a narrower temperature range encompassing the phase-change region.

\section{Parallel Tempering}

Parallel tempering 33 is very closely related to the tandem and parallel walker implementations of Jwalking, 21.26 with the essential difference being that instead of the higher-temperature configuration being copied over the lower-temperature configuration whenever a jump is accepted, the two configurations are exchanged. This close similarity between parallel tempering and tandem J-walking makes it very easy to convert a tandem J-walking simulation program to a parallel tempering one. The parallel tempering method's configuration exchange ensures that it obeys detailed balance (provided that the simulation has been warmed up enough to be in the asymptotic region), so there is no need to run the extra Metropolis passes to break correlations between the higher-temperature and lowertemperature walkers whenever an exchange move is accepted, which is required for tandem J-walking. In addition to offering greater efficiency, the obeying of de- 
tailed balance allows parallel tempering simulations to vary the exchange-attempt frequency in temperature regions where the exchange-acceptance rate is too low. In J-walking simulations, the jump-attempt frequency was typically kept at 10\%, since larger values required even more extra Metropolis passes to be run whenever a jump was accepted to break the correlations, thereby offsetting the efficiency gains made by increasing the jump-attempt frequency in the first place.

As with J-walking, the number of temperatures used in a parallel tempering simulation and their spacing cannot be chosen arbitrarily, but must be selected to ensure that the exchanges are accepted sufficiently often. Generally, more temperature walkers and smaller spacing are required for the solid-liquid phase-change region, and for very low temperatures. Since the heat capacity curves as a functions of temperature typically had peaks in the solid-liquid phase-change regions whose location and height I wanted to determine accurately, the number of temperatures and their spacing was also affected by the need to properly represent the heat capacity curves with a sufficient density of points to allow for good interpolation. This latter requirement resulted in my choosing temperature spacings in the phase-change region that were small enough that the exchange acceptance ratios were typically quite high. For very low temperatures, the classical heat capacity curve is nearly linear, thus requiring fewer points to represent. However, the exchange acceptance ratio decreases rapidly at lower temperatures, requiring more temperature walkers. Since the overall computation time is nearly proportional to the number of walkers, I was able to reduce the number of lowertemperature walkers needed by increasing the exchangeattempt frequency $P_{X}$ at lower temperatures, according to the relation

$$
P_{X}(T)=\frac{P_{X}\left(T_{H}\right)}{1-\left(\frac{P_{X}\left(T_{L}\right)-P_{X}\left(T_{H}\right)}{P_{X}\left(T_{L}\right)}\right)\left(\frac{T_{H}-T}{T_{H}-T_{L}}\right)},
$$

where $P_{X}\left(T_{L}\right)=\left(1+3 P_{X}\left(T_{H}\right)\right) / 4$. This expression increases the lowest-temperature exchange-attempt frequency $P_{X}\left(T_{L}\right)$ by a quarter of the range above the baseline highest-temperature exchange-attempt frequency, $P_{X}\left(T_{H}\right)$, which was set to $10 \%$.

The parallel tempering simulations done in this study were similar to those done by Neirotti et al. for $\mathrm{LJ}_{38} .32$ For each cluster simulation, a number of random walkers at various temperatures were run in parallel, with each temperature walker occasionally attempting an exchange move with the next higher temperature walker, except for the highest temperature walker, which attempted no exchanges directly (but did exchange configurations according to attempts made by the next-highest temperature walker). Since I had the lowest-energy isomers for each cluster from the J-walking distribution quenches, for most of the clusters I initialized the temperature walkers by using the lowest-energy isomer for the lowesttemperature initial configuration, the next lowest-energy isomer for the next lowest-temperature initial configuration, and so on. For the other clusters, I just used the lowest-energy isomer to initialize all of the initial configurations. It did not seem to matter which initialization scheme was used, and of course, random configurations could have been used, but at the cost of longer warmup periods. Because I initialized the simulations from the lowest-energy isomers, I could quickly run preliminary simulations that gave qualitatively good results, which I could use to fine-tune the number of parallel tempering temperature walkers and their spacing. These preliminary parallel tempering runs consisted of $10^{5}$ Metropolis warmup passes for each temperature walker, followed by $10^{5}$ parallel tempering warmup passes and then by 10 parallel tempering walks with preliminary data accumulation, with each walk being $10^{5}$ passes in length. The configurations for each temperature walker were saved at the end of the warmup period, and at the end of each walk. The heat capacity curves for each walk were examined for consistency, and if significant variations were found, the parallel tempering run was repeated (without the Metropolis warmup passes) from the last saved configurations, so that in effect, all of the preceding parallel tempering runs served as warmup runs for the final run. For some of the clusters, only parallel tempering warmup passes were run, indicating that the preceding Metropolis warmup passes were likely not necessary (although their computational expense was minor). After the preliminary runs appeared to be sufficiently warmed up, a longer parallel tempering run of $10^{5}$ warmup passes followed by $10^{7}$ total passes of data accumulation per temperature point was run (10 walks of $10^{6}$ passes each). Again, the heat capacity curves for each walk were checked for consistency; for a few clusters, the variations in the curves were still too large, and so the run was repeated from the last saved configurations. For $\mathrm{LJ}_{38}$, an especially long warmup was required, consistent with the findings of Neirotti et al 32

One important difference between my implementation of parallel tempering and that of Neirotti et al concerns the choice of the constraining radius $R_{c}$. Ideally, the value of the constraining radius should be large enough that the cluster properties being simulated are not adversely affected by the constraining potential, but not so large that cluster dissociations lead to overly long convergence times. In my previous study of clusters of size 4 to 24 , I used $R_{c}=4.0 \sigma$, a value large enough not to affect the heat capacity curves, except at higher temperatures, well past the solid-liquid phase-change regions. Thus, for this study, I also set $R_{c}=4.0 \sigma$ for the Metropolis and J-walker simulations. Neirotti et al used a value of $R_{c}=2.25 \sigma$ for their simulation of $\mathrm{LJ}_{38}$, since they had difficulties attaining ergodicity with larger constraining radii. For $\mathrm{LJ}_{38}$, such a small constraining radius was not a problem since the heat capacity peak occurred at a relatively low temperature. However, for most of the cluster sizes included in this study, such a small value would affect the heat capacity peak height and location. After 
some preliminary investigations, I chose to set $R_{c}=3.5 \sigma$ for parallel tempering simulations of cluster sizes 25 to 48 , and to $R_{c}=4.0 \sigma$ for the larger cluster sizes. Figure 1 illustrates the effects of the constraining radius on clusters $\mathrm{Ar}_{30}$ and $\mathrm{Ar}_{32}$. The heat capacity peak for $R_{c}=3.0 \sigma$ is slightly truncated, but the peak for $R_{c}=3.5 \sigma$ is in good agreement with the $R_{c}=4.0 \sigma$ peak, even though the high-temperature side of the peak is substantially affected. These two examples also show how well parallel tempering was able to overcome the quasiergodicity evident in the Metropolis curves. The two parallel tempering curves for $\mathrm{Ar}_{32}$ show very good agreement for the smaller peak, which is completely absent in the Metropolis simulations.

The converged heat capacity curve I obtained for $\mathrm{Ar}_{38}$ was mostly similar to the curve obtained byNeirotti et al. in their parallel tempering study of $\mathrm{LJ}_{38}, 32$ with a small shoulder in the low-temperature side of the peak. The reduced peak temperature 0.1653 was also in good agreement with that found by Neirotti et al. (0.166), although my peak value was slightly higher $\left(\left\langle C_{V}\right\rangle / N k_{B}=5.858\right.$ compared to 5.62) and the higher-temperature part of my curve rose substantially higher; these discrepancies are mostly due to Neirotti et al. having used the smaller constraining radius. As pointed out by Neirotti et al., the shoulder in the heat capacity curve is in contrast to the small lower-temperature peak found by Doyle, Wales and Miller in their study of $\mathrm{LJ}_{38} 33$ (their larger peak location and height were similar to the parallel tempering results, though, at 0.17 and 5.68, respectively).

Finally, follow-up parallel tempering simulations of $10^{6}$ passes per temperature were run for each cluster with periodic quenching of the configurations to determine the population distributions of the lower-energy isomers as functions of temperature. These were initialized using the final configurations from their corresponding preceding parallel tempering runs. Since I was primarily interested in using the quench results to provide qualitative insight into the heat capacity results, rather than determining the quench curves themselves with high accuracy, these shorter walk lengths were sufficient.

\section{RESULTS}

\section{A. Structural properties}

Much of the magic number behavior of cluster heat capacities can be understood in terms of the magic number behavior of the underlying potential surfaces For example, for the small clusters I studied earlier,11 the largest heat capacity peaks were observed for sizes $N=13,19$ and 23, corresponding to single, double and triple icosahedra, respectively. The same magic numbers were found in some corresponding structural properties as well, such as the binding energy differences,

$$
\Delta E_{b}(N)=-\left[V_{\min }(N)-V_{\min }(N-1)\right]
$$

The lowest-energy configurations for each of the clusters I examined in this study were obtained from quenches of J-walker distributions. These were consistent with the lowest-energy configurations found in other studies.4.33 From $N=25$ to 30 , the global minima all consisted of a $N=19$ double icosahedral core with atoms added to tetrahedrally bonded face sites (anti-Mackay overlayers), while for $N=31$ to 54 (except for $N=38$ ), the added atoms occupied sites corresponding to the outer shell of the $N=55$ Mackay icosahedron (Mackay overlayers); 9 $N=38$ did not fit the pattern, it being a truncated octahedron instead. 32 , 33 Clusters for $N=56$ to 60 had added atoms that contributed to the anti-Mackay overlayer of the 55-atom core, beginning the building up of the next Mackay icosahedron $(N=147)$.

Although the lowest-energy isomers play a significant role in cluster thermodynamics, other low-energy isomers also play important roles, and the size of the gap between the lowest-energy isomer and the next lowestenergy isomer is an especially important predictor of magic number behavior.26 6 Figure 2 shows the energy levels of the lower-energy configurations for each cluster size, relative to their global minimum. Sizes $N=$ $26,29,32,36,39,43,46,49,55$ and 58 have relatively large gaps between their global minimum and next higher minimum, compared to their immediate neighbors, and so can be considered a magic number sequence; the gaps between the lowest-energy and next lowest-energy isomers are also plotted in Figure 3 as functions of $N$. The Mackay icosahedron $N=55$ has an especially large gap. There is also much variation in the densities of the lowlying isomers evident in Figure 2, with densities being substantially greater for sizes $N=28$ to 37 than for the other sizes, while sizes $N=52$ to 56 have particularly low densities.

The binding energy differences for the clusters are also displayed in Figure 3. Except for $N=38$, the maxima in the binding energy differences are the same as the maxima in the energy differences between the two lowest-energy isomers. Also shown in Figure 3 is a plot of another indicator of structural magic number behavior, the second finite difference of the energy,

$\Delta_{2} E(N)=V_{\min }(N+1)+V_{\min }(N-1)-2 V_{\min }(N)$.

These magic numbers are also in agreement with the magic numbers obtained for the binding energy differences, again, except for $N=38$. The discrepancy in the magic number sequence for $N=38$ is due to that cluster being unique in this range by having an fcc structure as its global minimum, so that those measures that are relative to the cluster's immediate neighbors are considerably different than those of the other clusters, whose immediate neighbors are structurally similar.

Figure (1) shows a generalization of the zerotemperature $\Delta_{2} E(N)$ curve shown in Figure 3, with the curves extended to non-zero temperatures to form a $\Delta_{2} E(N, T)$ surface. The curves were obtained from 
parallel tempering simulations; the temperature scale is for Ar. The surface can be seen to encompass two general regions: a flat region at higher temperatures, corresponding to the clusters' liquid-like regions, and a corrugated region at lower temperatures, corresponding to the clusters' solid-like regions. The plot shows that many of the clusters are locked in their lowest-energy isomers for extended temperature ranges, especially the magic number sizes $N=26,29,39,43,46,49$ and 55 - the $\Delta_{2} E$ peak values differ very little from their zero-temperature values well into the phase-change region, until their abatement over a relatively narrow temperature range. The plot also shows that the phase-change temperature regions have a strong dependence on the cluster size, with the melting temperature generally decreasing from about $30 \mathrm{~K}$ for $N=26$ to about $20 \mathrm{~K}$ for $N=36$, and then generally increasing to a maximum of about $40 \mathrm{~K}$ for $N=55$.

\section{B. Thermodynamic properties}

Figure 5 plots the reduced potential energy per particle for each cluster size over a temperature range spanning the solid-like and liquid-like regions. Magic number behavior can be seen for sizes $N=26,29,32,36,39,43$, 46,49 and 55 by the relatively large differences at lower temperatures between their potential energies and those of their preceding cluster size. The Mackay icosahedron $N=55$ is so stable relative to its neighbors that its curve actually crosses several of the other curves. The phase regions are apparent in Figure 5 by the transition from the irregular spacing between the curves seen for the lower temperature, solid-like regions, to the regular spacing seen for the higher temperature, liquid-like regions. As was seen in the $\Delta_{2} E(N, T)$ surface in Figure 4, the melting temperature generally decreases as the cluster size increases from $N=25$, reaching a minimum of about $20 \mathrm{~K}$ for $N$ about 36 , and then increases to a maximum for $N=55$.

Curves of the reduced heat capacity per particle for each cluster size are shown in Figures 6, 7 and 8, while Figure 9 shows the corresponding peak height and temperature as functions of the cluster size; the peak parameters are also listed in Table I. The peak parameters were obtained by smoothing and interpolating the combined parallel tempering data (10 walks of $10^{6}$ passes per temperature point each). The associated parameter uncertainties were estimated by determining the peak height and location for each of the 10 walks separately, and calculating the standard error of the set of these values. As a check, additional runs of $10^{7}$ total passes per temperature were done for a few of the clusters, and the standard deviations of the resulting set of peak parameters were compared to the corresponding uncertainty estimates. These were found to be in good agreement. The extra check runs also showed the parallel tempering heat capacity results to be very reproducible, unlike the corresponding J-walking results.

Each of the clusters had at least one peak in its heat capacity curve, and overall, while their was much variation in the curves, systematic trends are evident. Cluster sizes $N=36,39,43,46,49$ and 55 had peak heights that were substantially greater than those of their immediate neighbors, and thus represent the magic numbers for the heat capacity sequence. These cluster sizes were also magic numbers for the $\Delta_{2} E(N)$ sequence and the lowest-energy isomer gaps shown in Figure 3 , but the other structural magic numbers $(N=26,29$ and 32$)$ did not show magic number behavior in their heat capacity curves. Instead, for cluster sizes 25 to 36 , both the heat capacity peak height and the peak temperature showed remarkably smooth variation, with the peak temperature slowly increasing, while the peak height slowly decreased in a nearly linear fashion, until the peak disappeared at $N=37$. As can be seen in Figure 6, this behavior was somewhat affected by the choice of the constraining radius. For the Metropolis simulations run at $R_{c}=4.0 \sigma$, the heat capacity curves had greater slopes at higher temperatures, resulting in the peak disappearing by $N=34$.

More interesting was the emergence and evolution of a second heat capacity peak, as the first peak gradually disappeared. Beginning with $N=30$, a small, lowertemperature peak can be seen in Figure 6 to develop and gradually increase in both height and temperature, until it becomes the dominant peak at $N=36$. This trend can be seen to continue in Figures 7 and 8, until the peak reaches its greatest height and highest temperature for the magic number size $N=55$. This behavior suggests partitioning the heat capacity peaks into two sequences, with the first sequence containing the small- $N$ peaks that evolve into the large, broad high-temperature peaks as the second Mackay shell initially builds up, while the second sequence originates from the smaller, narrower, lower-temperature peaks that evolve into the dominant peaks as the second Mackay shell is completed. The possible beginning of a third sequence corresponding to the initial building up of the third Mackay shell can be inferred from the curves in Figure 8, where $\mathrm{Ar}_{58}$ has a small, lower-temperature peak, and $\operatorname{Ar}_{59}$ and $\operatorname{Ar}_{60}$ have discernable shoulders in the low-temperature sides of their peaks.

The emergence of the smaller peak nearly coincides with the transition at $N=31$ from the anti-Mackay overlayer in the lowest-energy configuration to the Mackay overlayer, and the cluster sizes where two prominent heat capacity peaks are evident correspond to those sizes having the greatest density of low-lying isomers (Figure 2). Doye et al. found that for clusters in this size range, the high density of isomers was due to the small differences in energy between the various configuration types, antiMackay, Mackay, decahedral, fcc, etc; 10 similar findings were obtained for clusters bound by a Morse potential whose range parameter is similar to the Lennard-Jones potential.e

The two heat capacity sequences make the determi- 
nation of the cluster melting temperature from heat capacity peak temperatures ambiguous for sizes $N=31$ to 36 . Peaks in heat capacity curves have typically been associated with changes, particularly solid-liquid changes.20.25.32,33.35.39 However, peaks can arise from ther circumstances as well, such as mixing anomalies 23.24 and premelting effects, which can make the assignment of the cluster melting temperatures difficult. Calvo and Spiegelmann defined the melting temperatures of the sodium clusters they studied whose heat capacity curves had multiple peaks, as the temperature associated with the highest peak. 39 Applying such a definition to the clusters shown in Figure 6 that have two heat capacity peaks would imply that the reduced melting temperature would jump from $T^{*}=0.3784$ for $N=35$ to 0.1427 for $N=36$. Given the structural similarities of these two clusters, such a large difference in the melting temperature would be surprising, and as will be seen later when other cluster properties are examined, the two clusters do in fact have similar melting temperatures. Also, the potential energy curves shown in Figure 5 and the $\Delta_{2} E(N, T)$ surface shown in Figure 1 both suggest that the melting temperature for these clusters is about $T^{*}=0.17$, and thus intermediate to the two heat capacity peak temperatures.

There is much evidence of systematic error due to quasiergodicity in the Metropolis results, especially for sizes $N=31$ to 38 , where the Metropolis heat capacity curves had lower-temperature peaks that were either missing $(N=31$ to 34$)$ or poorly formed $(N=35$ to 38). For the other cluster sizes, the Metropolis heat capacity curves showed very good agreement with the parallel tempering results, except for temperatures near the heat capacity peak, where the Metropolis results for $10^{7}$ total passes per temperature point were substantially lower than the parallel tempering results, and even the Metropolis results for $10^{8}$ total passes were typically slightly lower. The fact that parallel tempering simulations of $10^{7}$ passes could provide significantly better results than similar Metropolis simulations of $10^{8}$ passes, for not much more computational overhead than Metropolis simulations of $10^{7}$ passes, clearly shows the superiority of parallel tempering over the Metropolis method.

There have been several previops studies of 55-atom cluster heat capacities done.20 $25.33,35-37$ The study most directly comparable to the parallel tempering results I obtained for $N=55$ is a $\mathrm{J}$-walking study of $\operatorname{Ar}_{55}$ done by López.25 He obtained peak parameters of $\left\langle C_{V}\right\rangle / N k_{B}=$ 19.5 and $T^{*}=0.29$, compared to my parallel tempering results of 17.45 and 0.294 , respectively. López used the same constraining volume radius as I did for $N=55$, $R_{c}=4.0 \sigma$, but his J-walker distributions consisted of only $4 \times 10^{4}$ configurations, which are considerably fewer than the $2.5 \times 10^{6}$ configurations I used for my J-walking studies of cluster sizes $N=25$ to 38 . Given the poor results I obtained with J-walking using larger distributions for smaller cluster sizes, I suspect that the discrepancy in the heat capacity peak parameters is due to López having used insufficiently representative J-walker distributions in his study. The heat capacity curve obtained from a Metropolis simulation of $10^{8}$ total passes per temperature that is shown in Figure 8 had peak parameters of 17.00 and 0.294 , respectively, which are close to the parallel tempering results. The small discrepancy between these Metropolis results and the parallel tempering results is similar to the discrepancies obtained for the other clusters of similar size. Labastie and Whetten obtained peak parameters of 15.4 and 0.3 for $\mathrm{Ar}_{55}$ using histogram methods, but their smaller peak height was most likely due to their constraining radius being considerably smaller at $R_{c}=2.6 \sigma$. Doye and Wales obtained peak parameters of 174 and 0.298 for $\mathrm{Ar}_{55}$ using the superposition method, 33,35 which is in very good agreement with my results.

\section{Bond length fluctuations}

The rms bond length fluctuation $\delta$ is another measure that has been used often in cluster simulations to estimate the melting temperature. the Lindemann criterion, melting occurs in bulk matter when $\delta$ is about $10 \%$ or greater. However, much care must be exercised in interpreting rms bond length fluctuations obtained from cluster simulations. First, as Calvo and Spiegelmann have pointed out, the Lindemann criterion was originally proposed for bulk systems, implying that it does not necessarily extend to finite clusters, and the choice of $10 \%$ for the critical value is somewhat arbitrary 39 Second, $\delta(T)$ is dependent on the simulation walk length, with the sharp rise in the curve generally occurring at lower temperatures as the walk length is increased.11 For cluster simulations of very long length, a value of $\delta \approx 0.1$ can be obtained at temperatures where the cluster spends most of its time in some solid-like form, with only occasional isomerizations to other solid-like forms, rather than undergoing the ready diffusion typical of liquid-like behavior. Because $\delta$ is obtained from averages over entire Metropolis walks or molecular dynamics trajectories, it cannot be estimated from simulations where configuration scrambling occurs, such as J-walking and parallel tempering, and so quasiergodicity issues further complicate matters. Using very long walk-lengths to reduce systematic errors arising from quasiergodicity can result in $\delta(T)$ curves whose transitions occur well before the melting phase-change.

Despite these caveats, rms bond length fluctuation curves have qualitative validity and can provide useful insights, especially in conjunction with other cluster properties. Curves of $\delta(T)$ for each cluster size are shown in Figures 10, 11 and 12 for averaged walk-lengths of $10^{6}$ and $10^{7}$ passes per temperature, while Figure 13 shows the $N$-dependence of the temperatures corresponding to the Lindemann-like threshold $\delta=0.2$. The threshold 
temperatures were estimated by smoothing and interpolating the $\delta(T)$ curves, and then searching for the interpolated temperature corresponding to $\delta=0.2$. I chose to set the threshold slightly higher than the usual Lindemann criterion of $\delta=0.1$, since some of the $\delta(T)$ curves had too much noise near $\delta=0.1$ to obtain a reliable estimate of the temperature corresponding to that threshold, and because the threshold is somewhat arbitrary anyway; the value $\delta=0.2$ was also typically near the inflection point of the smoothed curves' transition regions.

The $\delta(T)$ curves shown in Figures 10 to 12 are mostly similar, but there are some interesting differences. Generally, the steepness of the rise in $\delta$ in the transition region increases with cluster size, with the magic number sizes $N=43,46$ and 55 having especially sharp rises. Evidence of quasiergodicity can be clearly seen in some curves: the $\delta(T)$ curves for $N=31$ each have a noisy shoulder just before the transition region, while the curves for $N=32$ each have a small peak; the curves for $N=38$ show the cluster to be locked in its global minimum for all of the lower-temperature Metropolis simulations of length $10^{7}$ passes or fewer, until finally escaping at $T=19 \mathrm{~K}$. Evidence of pre-melting in the anti-Mackay overlayer of the third Mackay shell can be seen in sizes $N=56$ to 60 by the emergence and evolution of a shoulder in the lower-temperature part of the $\delta(T)$ curves.

Also indicated on the temperature axes in Figures 10 to 12 are the corresponding heat capacity peak temperatures obtained from the parallel tempering simulations (likewise, Figures 6 to 8 have the corresponding temperatures associated with the Lindemann-like threshold of $\delta=0.2$ indicated on their axes). These clearly show that the heat capacity peaks do not necessarily correspond to a cluster phase change. For cluster sizes $N=25$ to 29 , the heat capacity peak temperatures are well within the liquid-like region, according to the $\delta(T)$ curves, while for cluster sizes $N=30$ to 36 , where two heat capacity peaks are evident, the higher-temperature heat capacity peak is also clearly in the liquid-like region. Moreover, these temperatures are high enough that quasiergodicity in these regions of the $\delta(T)$ curves is not an issue. Likewise, most of the lower-temperature heat capacity peaks are found to be in the solid-like region of the $\delta(T)$ curves, well before the transition region (although ergodicity issues regarding the $\delta(T)$ curves in these regions could be involved). Only for sizes $N=35$ and $N=36$, are the lower-temperature heat capacity peak temperatures similar to the $\delta(T)$ transition regions. For the remaining cluster sizes shown in Figures 11 and 12, however, the heat capacity peak temperatures correspond quite closely to the end of the $\delta(T)$ transition regions, where the curves quickly change slope to mark the start of the liquid-like region.

Magic number behavior in the rms bond length fluctuations is evident in Figure 13, especially for the threshold temperature values obtained from the longer Metropolis simulations of $10^{7}$ passes. The threshold temperatures are significantly higher for the magic number sizes
$N=26,29,32,36,39,43,46,49$ and 55 , relative to their immediate neighbors. These are the same magic numbers seen in Figure 3 for $\Delta_{2} E(N)$ and for the energy gaps between the lowest-energy and next lowest-energy isomers, except for $N=58$. The $\delta$ data also account for the absence of magic number behavior in the heat capacities for sizes $N=26,29$ and 32. Since the large, broad, highertemperature heat capacity peaks seen slowly diminishing in Figures 3 and 6 are located well past the cluster solid-liquid phase-change regions, whatever magic number effects might exist for $N=26,29$ and 32 are masked by the systematic evolution of the heat capacity peak itself with increasing cluster size. Classical cluster heat capacities are inherently a measure of the width of the potential energy distributions. Typically, for temperatures near the cluster melting temperature, the distributions are much wider than those at lower temperatures (which span mostly lower-energy, solid-like forms) and those at higher temperatures (which span mostly higherenergy, liquid-like forms), since these distributions span both types of forms. However, the distribution width in the liquid region also typically increases with temperature, since the number of accessible configurations also increases. Thus, depending on the energy distribution of the higher-energy isomer potential minima, the inverse dependence of the heat capacity on temperature in Eq. (3) could result in a peak occurring in the liquidlike region of the curve that would have little relation to the cluster's solid-liquid phase change.

The threshold temperatures corresponding to $\delta=0.2$ in Figure 13 also imply that the cluster melting temperature generally decreases as the cluster size increases from $N=25$, reaching a minimum near $N=37$, then generally increases with cluster size, reaching a maximum for $N=55$, and decreases thereafter; magic number effects superimposed on these general trends account for the local variations. These general trends are consistent with those inferred from the $\Delta_{2} E(N, T)$ surface in Figure 4 and the potential energy curves in Figure 5. Casero and Soler calculated melting temperatures for LJ clusters of size $N=4$ to 34 in the microcannonical ensemble, defining the melting temperature to be the temperature where the free energies of the solid and liquid clusters become equal.12 Their results indicate a generally increasing trend with cluster size, with reduced melting temperatures for sizes $N=25$ to 34 all above 0.3 . These results are very similar to the temperatures seen for higher-temperature heat capacity peak temperatures in Figure 9, and thus are contrary to the melting temperatures implied by the rms bond length fluctuation data shown in Figure 13. Since the melting temperatures deduced from the $\delta$ data are in good agreement with the melting temperatures obtained from the heat capacity peak temperatures for cluster sizes greater than about $N=35$, and these temperatures are well below 0.3 for $N<40$, it is likely that the reduced melting temperatures for sizes $N=25$ to 34 are also well below 0.3 . 


\section{Quench results}

The temperature dependence on the distribution of quenched isomers is shown in Figure 14 for those Ar clusters whose heat capacities showed magic number behavior, and in Figures 15 to 17 for some selected non-magic number Ar clusters. Each plot shows the percentage of quenches to each of the three lowest-energy isomers, as well as the total percentage of quenches to the remaining higher-energy isomers; the data for all of these plots were all obtained from parallel tempering simulations. Much of the behavior exhibited by these curves can be rationalized in terms of the energy spacings and densities of the lower-energy isomers shown in Figure 2.

The plots for the magic number sizes are all very similar in form, with quenches in the lower-temperature, solid-like region represented solely by the lowest-energy isomer, and quenches to the other isomers only appearing at temperatures near the phase-change region, and higher. This is a consequence of the large gap between the lowest-energy and next lowest-energy isomers that is typical of magic number clusters. The only significant differences between the different cluster sizes is the temperature where the fraction of quenches to the lowestenergy isomer drops off substantially, which increases with cluster size, and can be seen to be very similar to the heat capacity peak temperature.

The plots for some non-magic cluster sizes shown in Figure $\overline{15}$ for $N<35$ are considerably different. The curves for $N=25$ have a similar form to the magic number curves in Figure 14, but both the decrease in the fraction of quenches to the lowest-energy isomer and the increase in the fraction of quenches to the higherenergy isomers are much more gradual. This gradual transition is consistent with the more gradual transition for the $\delta(T)$ curve, seen in Figure 10. Also, the transition region occurs well below the heat capacity peak temperature (but near the transition region indicated by $\delta(T))$. $\mathrm{Ar}_{27}$ has two very closely spaced lowest-energy isomers that are well below its next-higher energy isomers, and most of the lower-temperature quenches are to the second lowest-energy isomer. The temperature where the fraction of quenches to the second lowest-energy isomer exceeds the fraction to the lowest-energy isomer is similar to the peak temperature of the very small lowertemperature heat capacity peak. Again, the temperature region where the fraction of quenches to the higherenergy isomers increases substantially corresponds well to the transition region in the $\delta(T)$ curves, and is well below the higher-temperature heat capacity peak temperature. The quench curves for $N=31$ and 32 are similar to the $N=27$ curve, with the fraction of quenches to the lowest-energy isomer dropping rapidly at very low temperatures corresponding closely to the small heat capacity peak temperatures, and the fraction of quenches to higher-energy isomers gradually increasing at temperatures well below the peak temperature of the larger, higher-temperature heat capacity peaks. For $\mathrm{Ar}_{31}$, the density of lower-energy isomers is so great that quenches to higher-energy isomers dominate at temperatures even below the transition region for the $\delta(T)$ curves shown in Figure 10. The lower-temperature shoulders appearing in the $N=31 \delta(T)$ curves and the behavior of the corresponding quench curves are indicative of pre-melting and an extended phase-change region. $\mathrm{Ar}_{32}$ is a magic number according to such structural properties as $\Delta E_{b}(N)$ and $\Delta_{2} E(N)$, but not according to its heat capacity peak parameters. Its quench curves are also different than the typical magic-number quench curves seen in Figure 14. The $N=32 \delta(T)$ curves show unusual peaks in the solid region, just before their sharp rise. This region just follows the lower-temperature heat capacity peak, and according to the quench results, almost all of the quenches are to the second lowest-energy isomer. This indicates that the peaks in the $\delta(T)$ curves are a result of quasiergodicity (the Metropolis heat capacity curves in Figure 6 also show small, spurious peaks in this region). The quench curves for $N=33$ and 34 indicate easy access to the other low-lying isomers at low temperatures near the small heat capacity peak temperatures.

Figure 16 shows quench results for some non-magic number clusters for $N=37$ to 48. $\mathrm{Ar}_{37}$, like $\mathrm{Ar}_{31}$ and $\mathrm{Ar}_{34}$, has an especially high density of low-lying isomers, and its quench curves also show a substantial fraction of quenches to these isomers at temperatures corresponding to the heat capacity peak temperature. For $N=38$, the temperature region where the fraction of quenches to the lowest-energy fcc isomer drops off and the fraction of quenches to the lowest-energy icosahedral-like isomer (the second lowest-energy isomer) increases corresponds to the region just before the heat-capacity peak where a shoulder can be seen in Figme 7, which is consistent with other studies of this cluster 32 The quench curves for $N=$ 41,45 and 48 are similar to those for $N=37$, since these clusters all have accessible lower-energy isomers that are only slightly higher than the lowest-energy isomer, but the curves for $N=42$ are similar to the curves for the magic number clusters in Figure 14, indicating that its low-lying isomers are not very accessible.

The isomer energy levels for $N=50$ are similar to those for $N=45$ and 48 , and the corresponding quench curves shown in Figure 17 are likewise similar to those for $N=45$ and 48. $\operatorname{Ar}_{52}$ and $\operatorname{Ar}_{53}$ have some isomers having closely spaced potential energies only slightly above the lowest-energy isomer, and then very few isomers until $\Delta V_{\min } \approx 2.5$. This type of isomer energy distribution is similar to the distributions seen for binary Ar-Kr clusters, where many mixed isomer configurations had energies similar to the lowest-energy isomer 24 and the quench results are similar too, with the low-lying isomer quench curves showing plateaus throughout much of the solid-like temperature region. However, neither $\mathrm{Ar}_{52}$ nor $\mathrm{Ar}_{53}$ exhibited the small, very low temperature heat capacity peaks that were evident in the binary cluster curves. $\mathrm{Ar}_{56}$ has two low-energy isomers corresponding 
to the two unique ways a lone atom can be positioned on a 55-atom Mackay icosahedral core, separated by a large energy gap from the other isomers corresponding to rearrangements of the 55-atom core. The $N=56$ quench curves show substantial fractions of quenches to both isomers throughout much of the solid-like region until the phase-change region, when the higher-energy isomers become accessible. For $N=57$, there are several lower-energy isomers below $\Delta V_{\min } \approx 1.0$ that were represented in the quench distributions, but as with $N=52$ and 53, there was no corresponding lower-temperature heat capacity peak. Both the $N=57$ and 59 quench curves show evidence of premelting, consistent with their corresponding $\delta(T)$ curves in Figure 12 .

\section{CONCLUSIONS}

Compared to the irregularity with respect to cluster size $N$ that characterized the properties of the small clusters of size $N \leq 24$ that I studied previously, 11 the properties for the clusters sized $25 \leq N \leq 60$ showed more regularity and consistency. The lowest-energy isomers for these sizes were all dominated by icosahedral based geometries, except for $N=38$, whose lowest-energy isomer had fcc geometry (the lowest-energy icosahedral-like isomer for $N=38$ was only slightly less stable). For the icosahedral-like clusters, configurations having antiMackay overlayers were the lowest-energy isomers for $N<31$, while configurations having Mackay overlayers were the lowest-energy isomers for the remaining sizes up to $N=55$, after which the filling of the third shell began again with anti-Mackay overlayers. Correspondingly, the heat capacity peak parameters formed two overlapping sequences as functions of cluster size, with those clusters having anti-Mackay overlayer lowest-energy isomers showing a gradual evolution in their heat capacity peaks to higher temperature and smaller size, while those clusters having Mackay overlayer lowest-energy isomers had small, lower-temperature peaks that generally shifted to higher temperature and grew in size as $N$ increased.

For cluster sizes $25 \leq N \leq 35$, the heat capacity peak temperatures did not correspond well to the cluster melting temperature ranges inferred from the temperature dependence of other cluster properties, such as the rms bond length fluctuations and the distributions of quenched isomers. For each of the cluster sizes from $N=36$ to 60 , though, the melting temperature ranges obtained from the three measures were in qualitatively good agreement. The general trend in the cluster melting temperatures as a function of the cluster size over the range studied was an initial decrease, reaching a minimum near $N=36$, then an increase until a maximum at $N=55$, where the second Mackay shell was completed, followed by another decrease as atoms were added to the third Mackay shell. Superimposed on this general trend were local maxima corresponding to the magic numbers
$N=26,29,32,36,39,43,46,49$ and 55 . The heat capacity peak heights had a magic number series that was a subset of this sequence, with local maxima occuring at $N=36,39,43,46,49$ and 55; for the other magic number sizes where the heat capacity peaks did not show magic number behavior $(N=26,29$, and 32$)$, the heat capacity peaks were located well away from the solid-liquid phase-change regions.

The magic number sequence for the cluster melting temperatures coincided very well with the magic number sequences obtained for structural properties such as the binding energy difference $\Delta E_{b}(N)$, the second finite difference of the energy $\Delta_{2} E(N)$, and the gap between the lowest-energy and next lowest-energy isomers. The only differences were $N=38$ being a magic number for the $\Delta E_{b}(N)$ sequence instead of $N=39$, and $N=58$, which was a magic number for all three structural properties, but was not for either the heat capacity or the rms bond length fluctuation sequences. This similarity in the magic number sequences is consistent with the widely held view that the thermodynamic behavior of clusters in the phase-change region is dominated by their potential energy surfaces.

The parallel tempering method was found to be especially useful for overcoming quasiergodicity and accurately calculating the heat capacities of these clusters, particularly for the range $N=30$ to 38 , where the Metropolis method was hard pressed to provide even qualitatively correct results. Even the J-walking method, which had worked very well for smaller cluster sizes, was unable to determine heat capacities reproducibly for clusters in this range. Given also the relatively small computational overhead for the method compared to Metropolis Monte Carlo, parallel tempering currently stands as the method of choice for cluster Monte Carlo simulations.

\section{ACKNOWLEDGMENTS}

The support of the Natural Sciences and Engineering Research Council of Canada (NSERC) for part of this research is gratefully acknowledged. I thank Northern Digital, Inc. for generously providing me the use of their workstations for some of the calculations reported, and the University of Waterloo for the use of their facilities. I also thank David L. Freeman for helpful discussions.

${ }^{1}$ M. R. Hoare and P. Pal, Adv. Phys. 20, 161 (1971).

${ }^{2}$ R. S. Berry, T. L. Beck, H. L. Davis and J. Jellinek, in Advances in Chemical Physics, edited by I. Prigogine and S. A. Rice (Wiley, New York, 1988), Vol. 70B, p. 75.

${ }^{3}$ H. L. Davis, J. Jellinek, and R. S. Berry, J. Chem. Phys. 86, 6456 (1987). 
${ }^{4}$ T. L. Beck, J. Jellinek, and R. S. Berry, J. Chem. Phys. 87, 545 (1987).

${ }^{5}$ J. E. Adams and R. M. Stratt, J. Chem. Phys. 93, 1332 (1990).

${ }^{6}$ J. D. Honeycutt and H. C. Andersen, J. Phys. Chem. 91, 4950, (1987).

7 J. A. Northby, J. Chem. Phys. 87, 6166 (1987).

${ }^{8}$ D. G. Vlachos, L. D. Schmidt, and R. Aris, J. Chem. Phys. 96, 6880 (1992); 96, 6891 (1992).

9 J .P. K. Doye, D. J. Wales, and R. S. Berry, J. Chem. Phys. 103, 4234 (1995).

${ }^{10}$ J. P. K. Doye, M. A. Miller, and D. J. Wales, J. Chem. Phys. 111, 8417 (1999).

${ }^{11}$ D. D. Frantz, J. Chem. Phys. 102, 3747 (1995).

${ }^{12}$ R. Casero and J. M. Soler, J. Chem. Phys. 95, 2927 (1991).

13 T. Ikeshoji, B. Hafskjold, Y. Hashi, and Y. Kawazoe, J. Chem. Phys. 105, 5126 (1996).

${ }^{14}$ O. Echt, K. Sattler, and R. Recknagel, Phys. Rev. Lett. 47, 1121 (1981).

${ }^{15}$ E. K. Parks, B. J. Winter, T. D. Klots, and S. J. Riley, J. Chem. Phys. 94, 1882 (1991); E. K. Parks, K. P. Kearns, and S. .J Riley, J. Chem. Phys. 109, 10207 (1998).

${ }^{16}$ D. Prekas, C. Lüder, and M. Velegrakis, J. Chem. Phys. 108, 4450 (1998).

${ }^{17}$ M. Sakurai, K. Watanabe, K. Sumiyama, and K. Suzuki, J. Chem. Phys. 111, 235 (1999).

18 J. P. Valleau and S. G. Whittington, in Statistical Mechanics, edited by B. J. Berne (Plenum, New York, 1977), Ch. 4, p. 145.

${ }^{19}$ F. H. Stillinger and T. A. Weber, Phys. Rev. A 25, 978 (1982).

${ }^{20}$ P. Labastie and R. L. Whetten, Phys. Rev. Lett. 65, 1567 (1990).

${ }^{21}$ D. D. Frantz, D. L. Freeman, and J. D. Doll, J. Chem. Phys. 93, 2769 (1990).

${ }^{22}$ D. D. Frantz, D. L. Freeman, and J. D. Doll, J. Chem. Phys. 97, 5713 (1992).

${ }^{23}$ G. E. Lopez and D. L. Freeman, J. Chem. Phys. 98, 1428 (1993).

${ }^{24}$ D. D. Frantz, J. Chem. Phys. 105, 10030 (1996); J. Chem. Phys. 107, 1992 (1997).

${ }^{25}$ G. E. López, J. Chem. Phys. 104, 6650 (1996).

${ }^{26}$ A. Matro and D. L. Freeman, and R. Q. Topper, J. Chem. Phys. 104, 8690 (1996).

${ }^{27}$ E. Curotto, D. L. Freeman, and J. D. Doll, J. Chem. Phys. 109, 1643 (1998).

${ }^{28}$ H. Xu and B. J. Berne, J. Chem. Phys. 110, 10299 (1999).

${ }^{29}$ R. Zhou and B. J. Berne, J. Chem. Phys. 107, 9185 (1997).

${ }^{30}$ C. J. Geyer and E. A. Thompson, J. Am. Stat. Assoc. 90, 909 (1995).

${ }^{31}$ M. R. Ghayal and E. Curotto, J. Chem. Phys. 113, 4298 (2000).

32 J. P. Neirotti, F. Calvo, D. L. Freeman and J. D. Doll, J. Chem. Phys. 112, 10340 (2000); F. Calvo, J. P. Neirotti, D. L. Freeman and J. D. Doll, J. Chem. Phys. 112, 10350 (2000).

33 J. P. K. Doye, D. J. Wales and M. A. Miller, J. Chem. Phys. 109, 8143 (1998).

${ }^{34}$ J .P. K. Doye, M. A. Miller and D. J. Wales, J. Chem. Phys. 110, 6896 (1999).
35 J. P. K. Doye and D. J. Wales, J. Chem. Phys. 102, 9659 (1995).

${ }^{36}$ H. Cheng, X. Li, R. L. Whetten, and S. Berry, Phys. Rev. A 46, 791 (1992).

${ }^{37}$ V. V. Nauchitel and A. J. Pertsin, Mol. Phys. 40, 1341 (1980).

${ }^{38}$ C. Seko, and K. Takatsuka, J. Chem. Phys. 104, 8613 (1996).

${ }^{39}$ F. Calvo, and F. Spiegelmann, J. Chem. Phys. 112, 2888 (2000). 
TABLE I. Cluster heat capacity peak parameters for $25 \leq N \leq 60$. These values were obtained by smoothing and interpolating the parallel tempering data shown in Figs. 6, 6 and 8, which were based on the combined results of 10 walks, each consisting of $10^{6}$ passes of data accumulation per temperature. The uncertainties were estimated as the standard errors of the set of peak parameters that were obtained from the interpolated heat capacity curves for each of the 10 walks.

\begin{tabular}{|c|c|c|c|c|c|c|c|c|c|}
\hline \multirow[b]{2}{*}{$\mathrm{N}$} & \multicolumn{2}{|c|}{ Lower temperature peak } & \multicolumn{2}{|c|}{ Higher temperature peak } & \multirow[b]{2}{*}{$\mathrm{N}$} & \multicolumn{2}{|c|}{ Lower temperature peak } & \multicolumn{2}{|c|}{ Higher temperature peak } \\
\hline & $T^{*}$ & $\left\langle C_{V}^{*}\right\rangle / N$ & $T^{*}$ & $\left\langle C_{V}^{*}\right\rangle / N$ & & $T^{*}$ & $\left\langle C_{V}^{*}\right\rangle / N$ & $T^{*}$ & $\left\langle C_{V}^{*}\right\rangle / N$ \\
\hline 25 & & & $0.3134 \pm 0.0016$ & $6.425 \pm 0.011$ & 43 & & & $0.1970 \pm 0.0009$ & $7.815 \pm 0.032$ \\
\hline 26 & & & $0.3116 \pm 0.0015$ & $6.380 \pm 0.012$ & 44 & & & $0.1986 \pm 0.0009$ & $7.012 \pm 0.027$ \\
\hline 27 & $0.0183 \pm 0.0004$ & $2.965 \pm 0.001$ & $0.3157 \pm 0.0015$ & $6.313 \pm 0.011$ & 45 & & & $0.2051 \pm 0.0009$ & $7.682 \pm 0.029$ \\
\hline 28 & & & $0.3242 \pm 0.0016$ & $6.210 \pm 0.013$ & 46 & & & $0.2210 \pm 0.0006$ & $9.781 \pm 0.040$ \\
\hline 29 & & & $0.3299 \pm 0.0025$ & $6.092 \pm 0.011$ & 47 & & & $0.2204 \pm 0.0005$ & $8.681 \pm 0.033$ \\
\hline 30 & $0.0554 \pm 0.0010$ & $3.059 \pm 0.002$ & $0.3337 \pm 0.0020$ & $5.995 \pm 0.014$ & 48 & & & $0.2289 \pm 0.0010$ & $9.067 \pm 0.041$ \\
\hline 31 & $0.0279 \pm 0.0019$ & $3.443 \pm 0.079$ & $0.3396 \pm 0.0018$ & $5.888 \pm 0.013$ & 49 & & & $0.2374 \pm 0.0006$ & $10.257 \pm 0.089$ \\
\hline 32 & $0.0603 \pm 0.0008$ & $4.115 \pm 0.035$ & $0.3484 \pm 0.0023$ & $5.814 \pm 0.009$ & 50 & & & $0.2472 \pm 0.0006$ & $9.702 \pm 0.047$ \\
\hline 33 & $0.0757 \pm 0.0015$ & $4.336 \pm 0.063$ & $0.3511 \pm 0.0033$ & $5.737 \pm 0.010$ & 51 & & & $0.2615 \pm 0.0003$ & $11.531 \pm 0.045$ \\
\hline 34 & $0.0612 \pm 0.0011$ & $4.202 \pm 0.024$ & $0.3641 \pm 0.0021$ & $5.656 \pm 0.005$ & 52 & & & $0.2743 \pm 0.0004$ & $13.460 \pm 0.061$ \\
\hline 35 & $0.1070 \pm 0.0014$ & $4.759 \pm 0.031$ & $0.3771 \pm 0.0064$ & $5.600 \pm 0.010$ & 53 & & & $0.2828 \pm 0.0005$ & $15.197 \pm 0.064$ \\
\hline 36 & $0.1415 \pm 0.0015$ & $5.715 \pm 0.067$ & $0.4097 \pm 0.0074$ & $5.600 \pm 0.006$ & 54 & & & $0.2897 \pm 0.0004$ & $16.766 \pm 0.104$ \\
\hline 37 & & & $0.1398 \pm 0.0017$ & $5.027 \pm 0.023$ & 55 & & & $0.2940 \pm 0.0005$ & $17.450 \pm 0.098$ \\
\hline 38 & & & $0.1653 \pm 0.0005$ & $5.858 \pm 0.030$ & 56 & & & $0.2929 \pm 0.0007$ & $15.280 \pm 0.131$ \\
\hline 39 & & & $0.1948 \pm 0.0009$ & $7.554 \pm 0.029$ & 57 & & & $0.2917 \pm 0.0005$ & $13.645 \pm 0.081$ \\
\hline 40 & & & $0.1938 \pm 0.0004$ & $7.250 \pm 0.021$ & 58 & $0.1844 \pm 0.0022$ & $3.721 \pm 0.005$ & $0.2871 \pm 0.0010$ & $11.702 \pm 0.033$ \\
\hline 41 & & & $0.1875 \pm 0.0011$ & $6.602 \pm 0.015$ & 59 & & & $0.2824 \pm 0.0006$ & $10.934 \pm 0.053$ \\
\hline 42 & & & $0.1884 \pm 0.0006$ & $6.828 \pm 0.025$ & 60 & & & $0.2786 \pm 0.0007$ & $10.340 \pm 0.073$ \\
\hline
\end{tabular}


FIG. 1. Effects of the constraining radius $R_{c}$ on cluster reduced heat capacities per particle for simulations of $\mathrm{Ar}_{30}$ (at left) and $\mathrm{Ar}_{32}$ (at right). The filled circles represent parallel tempering results for $R_{c}=3.0 \sigma$, while the squares represent parallel tempering results for $R_{c}=3.5 \sigma$; these were obtained from walks of $10^{7}$ total passes per temperature point. The error bars are two standard deviations on each side. The dotted line and the diamonds represent Metropolis results for $R_{c}=4.0 \sigma$, which were obtained from walks of $10^{7}$ and $10^{8}$ total passes per temperature point, respectively. The curves with different constraining radii are in very good agreement on the low-temperature side of the peak, but show substantial deviations on the high-temperature side of the peak, effecting the peak height and location slightly. The differences between the $R_{c}=3.5 \sigma$ and $R_{c}=4.0 \sigma$ peak height and location were minor in these two cases, and insignificant for most of the other cluster sizes whose parallel tempering results were obtained using $R_{c}=3.5 \sigma$. The smaller lower-temperature peaks seen in the parallel tempering curves are absent in the Metropolis curves, indicating the Metropolis simulations were subject to substantial quasiergodicity.

FIG. 2. Cluster local potential energy minima relative to the global minimum, in reduced units. Magic number behavior can be seen for $N=26,29,32,36,39,43,46,49$ and 55, which have large gaps between their global minimum energy and their next higher energy configurations, compared to their immediate neighbors. The potential energies were obtained from J-walking and parallel tempering quench studies.

FIG. 3. The binding energy difference as a function of cluster aggregate size (top plot), the second finite difference of the energy as a function of cluster size (middle plot), and the energy gap between the lowest-energy isomer and the next lowest-energy isomer, as a function of cluster size (bottom plot), all in reduced units. Except for $N=38$ and 39 , the three measures show the same peak values. The binding energy differences and the second finite differences for $5 \leq N \leq 24$ have been included for comparison, using data from Ref. 11.

FIG. 4. The second finite difference of the energy $\Delta_{2} E$ as a function of temperature $T$ and size $N$, for Ar clusters. The structure of the zero temperature curve $\Delta_{2} E(N)$ (also shown in Fig. 3) can be seen to extend well into the higher-temperature regions for the magic number sizes (seen as ridges in the surface), which indicates the persistence of their solid-like lowest-energy isomers over relatively large temperature ranges. The flat regions of the surface at higher temperature correspond to the cluster liquid-like regions, and so the phase-change regions correspond to the temperatures where the ridges abate.

FIG. 5. Cluster potential energies per particle as functions of temperature, obtained from parallel tempering simulations for clusters ranging from $N=25$ (top curve) to $N=60$ (bottom curve). Reduced units have been used; the absolute temperature scale at the top is for Ar. Magic number sizes for $N=26,29,32,36,39,43,46,49$ and 55 are indicated by the dotted curves, and correspond to those clusters having relatively large energy differences from their preceding cluster sizes at low temperatures. A rough indication of the phase-change region from solid-like to liquid-like can be ascertained by the change from the irregular spacing between the curves seen at lower temperatures to the regular spacing seen at higher temperatures.

FIG. 6. Reduced heat capacities per particle as functions of temperature for Ar clusters of size $N=25$ to 36 . The open circles and thicker solid line represent the results obtained from parallel tempering runs of $10^{7}$ passes per temperature point. The dotted line represents the results obtained from Metropolis runs of $10^{7}$ passes per temperature point, while the thin solid line represents the results obtained from similar Metropolis runs of $10^{8}$ passes per temperature point. Beginning with $\mathrm{Ar}_{30}$, a smaller, lower-temperature peak can be seen evolving in the parallel tempering curves and moving to higher temperatures, while the higher-temperature peak gradually disappears. Quasiergodicity is evident in the Metropolis results of these cluster sizes by the absent and malformed lower temperature peaks. As in Fig. 1, the discrepancies between the parallel tempering and Metropolis curves for higher temperatures are due to the parallel tempering simulations having been run with a smaller constraining radius of $3.5 \sigma$, instead of the value of $R_{c}=4.0 \sigma$ used in the Metropolis simulations. The large ticks on the temperature axes indicate the temperatures where the Metropolis rms bond length fluctuations rose sharply for walks of $10^{7}$ passes per temperature (higher temperature) and $10^{8}$ passes.

FIG. 7. Same as Fig. 6, but for clusters ranging in size from $N=37$ to 48 . Note the different heat capacity scale.

FIG. 8. Same as Fig. 6, but for clusters ranging in size from $N=49$ to 60 . For these sizes, the constraining radius was set to $4.0 \sigma$ for both the parallel tempering and Metropolis simulations. Again, note the different heat capacity scale. 
FIG. 9. Magic number behavior in cluster heat capacities. The upper plot shows the reduced heat capacity peak values as a function of the cluster size, while the lower plot shows the corresponding peak reduced temperatures as a function of the cluster size; the right-hand axis is representative of Ar. The peak parameters were obtained from interpolations of the parallel tempering data; parameter values for $N<25$ have been included from Ref. 11 for added context. Two general sequences have been identified as a consequence of the clusters in the range $30 \leq N \leq 37$ having two prominent peaks. The circles indicate the heat capacity parameters for the broader, higher temperature peaks, which have evolved from the solid-liquid phase-change region peaks of the smaller cluster sizes, while the squares represent the narrower, lower temperature peaks, which evolve into the solid-liquid phase-change region peaks of the larger cluster sizes. The triangles represent the parameters for the very small, broad peaks seen for sizes $N=27$ and 58. The magic number labels indicate the cluster sizes whose peaks are higher than those of their immediate neighbors.

FIG. 10. Root mean square bond length fluctuations as functions of the temperature for Ar clusters of size $N=25$ to 36 . The solid circles represent data obtained from 10 Metropolis walks, each consisting of $10^{6}$ passes per temperature point, while the open circles represent data obtained from 10 similar Metropolis walks of $10^{7}$ passes in length. The error bars are single standard deviations for the 10 walks. The large ticks on the temperature axes indicate the corresponding cluster heat capacity peak temperatures.

FIG. 11. Same as Fig. 10, but for clusters ranging in size from $N=37$ to 48.

FIG. 12. Same as Fig. 10, but for clusters ranging in size from $N=49$ to 60 .

FIG. 13. Magic number behavior in cluster rms bond length fluctuations. The curves plot the reduced temperatures associated with the Lindemann-like threshold $\delta=0.2$, as a function of cluster size. The squares represent the values corresponding to $\delta(T)$ curves whose data were obtained from Metropolis walks of $10^{6}$ passes per temperature point, while the circles represent values obtained from similar Metropolis simulations of $10^{7}$ passes. The right-hand axis is representative of Ar. The magic numbers are a subset of those obtained in Figure 3 for the second finite differences in the energy.

FIG. 14. Quenched isomer distribution curves for Ar clusters as functions of temperature for the magic number sizes $N=36,39,43,46,49$, and 55. The data were obtained from parallel tempering simulations of $10^{6}$ total passes per temperature. The filled circles represent the fraction of isomers that quenched to the lowest-energy isomer, the open circles the second lowest-energy isomer, and the squares the third lowest-energy isomer, while the line represents the sum of the remaining isomers. The dotted vertical lines in each plot indicate the corresponding heat capacity peak temperatures.

FIG. 15. Same as Figure 14, but for the non-magic number sizes $N=25,27$ and 31 to 34 .

FIG. 16. Same as Figure 14, but for the non-magic number sizes $N=37,38,41,42,45$ and 48.

FIG. 17. Same as Figure 14, but for the non-magic number sizes $N=50,52,53,56,57$ and 59 . 
This figure "figure1.gif" is available in "gif" format from: http://arxiv.org/ps/physics/0107013v1 
This figure "figure2.gif" is available in "gif" format from: http://arxiv.org/ps/physics/0107013v1 
This figure "figure3.gif" is available in "gif" format from: http://arxiv.org/ps/physics/0107013v1 
This figure "figure4.gif" is available in "gif" format from: http://arxiv.org/ps/physics/0107013v1 
This figure "figure5.gif" is available in "gif" format from: http://arxiv.org/ps/physics/0107013v1 
This figure "figure6.gif" is available in "gif" format from: http://arxiv.org/ps/physics/0107013v1 
This figure "figure7.gif" is available in "gif" format from: http://arxiv.org/ps/physics/0107013v1 
This figure "figure8.gif" is available in "gif" format from: http://arxiv.org/ps/physics/0107013v1 
This figure "figure9.gif" is available in "gif" format from: http://arxiv.org/ps/physics/0107013v1 
This figure "figure10.gif" is available in "gif" format from: http://arxiv.org/ps/physics/0107013v1 
This figure "figure11.gif" is available in "gif" format from: http://arxiv.org/ps/physics/0107013v1 
This figure "figure12.gif" is available in "gif" format from: http://arxiv.org/ps/physics/0107013v1 
This figure "figure13.gif" is available in "gif" format from: http://arxiv.org/ps/physics/0107013v1 
This figure "figure14.gif" is available in "gif" format from: http://arxiv.org/ps/physics/0107013v1 
This figure "figure15.gif" is available in "gif" format from: http://arxiv.org/ps/physics/0107013v1 
This figure "figure16.gif" is available in "gif" format from: http://arxiv.org/ps/physics/0107013v1 
This figure "figure17.gif" is available in "gif" format from: http://arxiv.org/ps/physics/0107013v1 